\title{
Volume Isotope Shifts in Low Lying Transitions of Au I
}

\author{
A. Rosén \\ Department of Physics, Chalmers University of Technology, Göteborg, Sweden \\ B. Fricke and G. Torbohm \\ Institut für Theoretische Physik, Universität Kassel, Federal Republic of Germany
}

Received November 30, 1983

\begin{abstract}
Electronic factors in the volume isotope shift have been calculated in an ab initio way with the relativistic Dirac-Fock method for a number of different optical single/and two-photon transitions in Au I. The agreement with a semi-empirical method is within $10 \%$ for the resonance transition. For this one and a few other transitions the effect of core excitation has been analyzed with the Multi-configuration Dirac-Fock method as well, and it was found to reduce the electronic factor in the order of $5 \%$.
\end{abstract}

Programs for measurements of nuclear spins, hyperfine structure constants, and isotope shifts (IS) for series of neutron-deficient as well as neutron-rich isotopes have been going on during the last years at the ISOLDE facility at CERN $[1-5]$. The objective of these investigations is to get information on the single particle structure of the investigated nuclei, to follow trends of nuclear properties for series of isotopes, and isotones as well as to test the validity of different nuclear models [6]. The measurements give the nuclear spins directly, while the evaluation of nuclear moments for a certain isotope, and change of nuclear charge radii between isotopes, requires knowledge of the electronic factor for that particular interaction [7].

The magnetic ground state hyperfine structure, and the nuclear spins have been determined for a number of radioactive Au-isotopes [8-10] by the atomicbeam magnetic-resonance method. Information about the quadrupole moment exists only for the stable isotope ${ }^{197} \mathrm{Au}[11]$. Recently [12-14] IS have been measured in the resonance line between ${ }^{197} \mathrm{Au}$ (stable), and ${ }^{195} \mathrm{Au}\left(T_{1 / 2}=183 d\right)$ isotopes at Mainz as a pilot project of systematic IS-measurements for a series of short-lived Au-isotopes at the ISOLDE facility. In this project measurements are planned also for some $6 s-n s$ two-photon transitions. Nuclear data properties, derived from these measurements, then are planned to be used for comparison with data for neighbouring isotopes of $\mathrm{Os}, \mathrm{Ir}, \mathrm{Pt}, \mathrm{Hg}, \mathrm{Tl}$, and $\mathrm{Pb}[5,6]$.
Recently, relativistic Multi-configuration Dirac-Fock (MCDF) wave functions, coupled to good angular momentum $J$, have been obtained for low lying states of $\mathrm{Ba}$ I and $\mathrm{Ba}$ II [15]. Good agreement was achieved with an electronic factor for the volume isotope shift, evaluated from isotope shifts in muonic $\mathrm{X}$-rays [16], although there is some discrepancy to more recently published muonic X-ray data [17]. The reason for this discrepancy is at present not understood. Further, a comparison of experimental slopes of lines, obtained by a King plot analysis, was found to be reproduced well by these wave functions. Also the $J$-dependence of the volume IS of the resonance transitions in Ba II was found to be in complete agreement with the experimental values $[18,19]$. These calculations are extended at present to all elements in group II a and IIb of the periodic table [20]. As a continuation of those calculations we present in this paper electronic factors for a number of transitions within the AuI spectrum, which will be useful in the analysis of data from the proposed IS-measurements.

The isotope shift in an atomic transition between two isotopes with mass $A$ and $A^{\prime}$ is the sum of field shift $\delta v_{f}$, and mass shift $\delta v_{m}[21]$

$\delta v^{A A^{\prime}}=\delta v_{f}^{A A^{\prime}}+\delta v_{m}^{A A^{\prime}}$

where the field shift is given as product of an electronic factor $F_{i}$, and a nuclear factor $\lambda$ proportional to $\delta\left\langle r^{2}\right\rangle^{A A^{\prime}}$ in first order, i.e.:

$\delta v_{f_{i}}^{A A^{\prime}}=F_{i} \cdot \lambda \approx F_{i} \delta\left\langle r^{2}\right\rangle^{A A^{\prime}}$. 


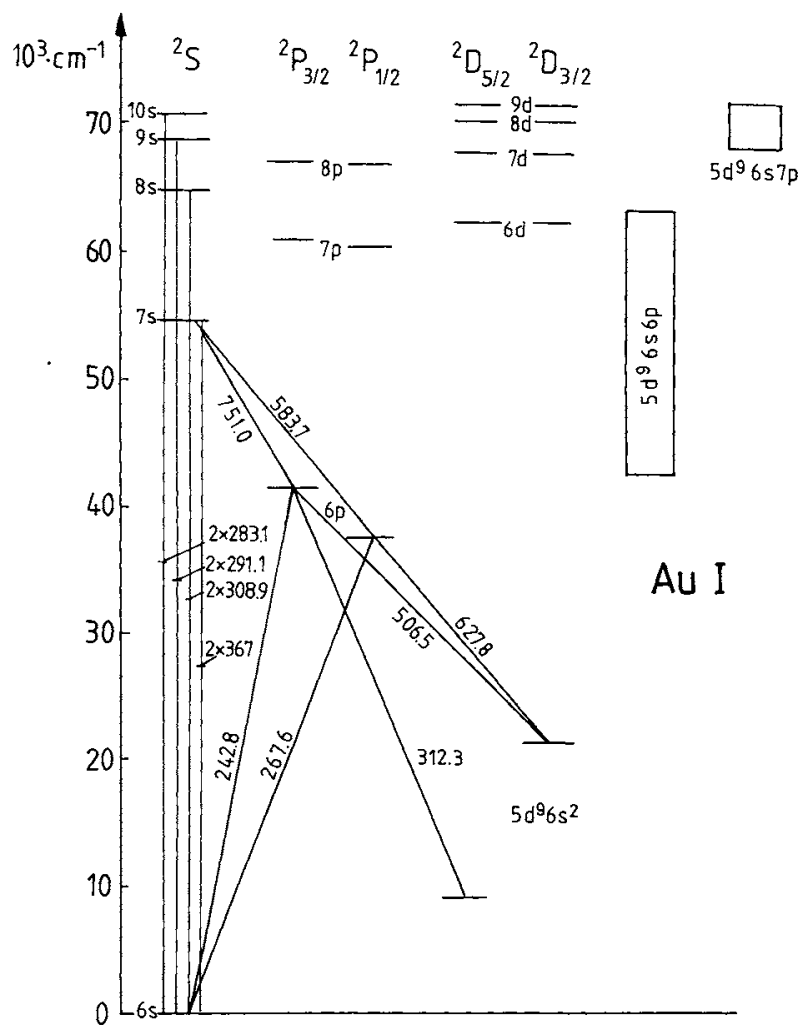

Fig. 1. Level energy diagram of the neutral Au atom. Wavelengths for a number of one-photon as well as two-photon $6 s^{2} S_{1 / 2}$ $-n s^{2} S_{1 / 2}$ transitions are indicated

The normal part of the mass shift can be evaluated exactly, while the specific part is usually estimated in some empirical way [22]. Since the specific mass shift is sensitive to correlation effects, an ab-initio evaluation requires extensive multi-configuration [23] or many-body calculations [24]. In this work we will calculate the volume isotope shift only, applying the relativistic MCDF-code by Desclaux [25].

A level energy diagram for the low lying states of Au I, as constructed from the energy levels of Moore [26], is presented in Fig. 1. The spectrum is alkalilike with a $6 s^{2} S_{1 / 2}$ ground state with a number of $n s^{2} S_{1 / 2}, n p^{2} P_{1 / 2}, n p^{2} P_{3 / 2}, n d^{2} D_{3 / 2}$, and $n d^{2} D_{5 / 2}$ Rydberg states. The lowest ${ }^{2} D_{3 / 2}$, and ${ }^{2} D_{5 / 2}$ states originate from the even parity $5 d^{9} 6 s^{2}$ configuration. Wavelengths for a number of one-photon as well as some two-photon $6 s^{2} S_{1 / 2}-n s^{2} S_{1 / 2}$ transitions are indicated. Separate single configuration Dirac-Fock calculations have been done for each state. Since Au is a heavy element, and the electronic charge density varies strongly over the nuclear volume, calculations were done with an extended nucleus using a twoparameter Fermi-nuclear charge distribution [27] with 21 mesh points inside the nucleus. The half-
Table 1. Electron charge density at the nucleus (at $r=0$ ) from single and multi-configuration calculation for various states in Au I and the ground state of Au II. The values are given relative to the ${ }^{2} S_{1 / 2}$ ground state

\begin{tabular}{ll}
\hline State & $4 \pi|\psi(0)|^{2}\left[(\text { a.u. })^{-3}\right]$ \\
\hline SC-calc. & MC-calc. \\
\hline
\end{tabular}

Au I with $5 d^{10}$-core

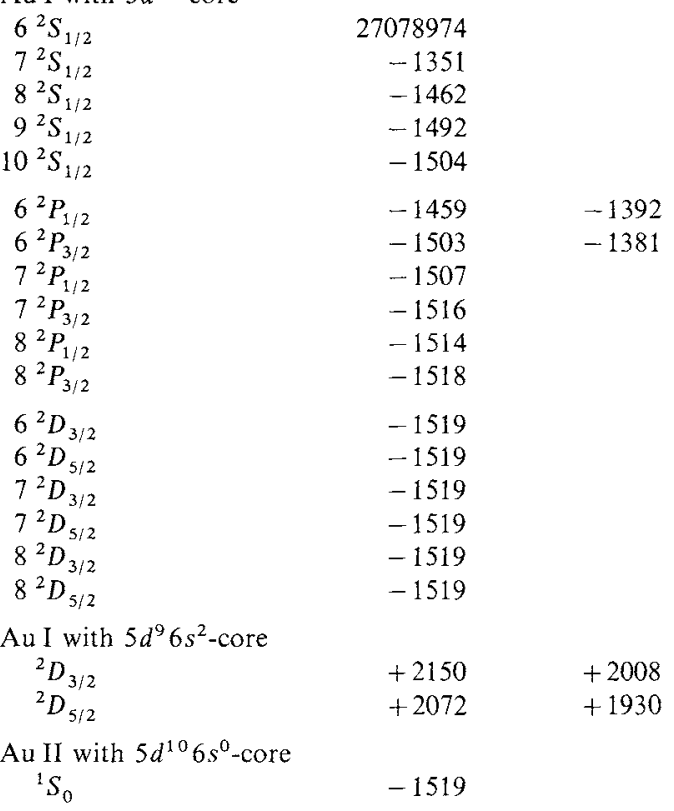

charge radius $R_{c}$, and thickness parameters $t$, were $R_{c}=6.38 \mathrm{fm}$, and $t=2.32 \mathrm{fm}$. The charge density at the nucleus (at $r=0$ ) was evaluated in each calculation (Table 1), and used for the calculation of electronic factors for the transitions given in Table 2.

We notice how the charge density at the nucleus for highly excited states approaches the value achieved for the ionized system Au II. Since the electronic charge density as well as the difference of the charge density between different configurations vary over the nuclear volume, the higher nuclear moments become effective in the evaluation of the parameter $\lambda$ in formula (2). Formula (2) reads more exactly [28]

$$
\begin{aligned}
\delta v_{f_{i}}^{A A^{\prime}} & =F_{i} \cdot \lambda=\left[c_{1} \delta\left\langle r^{2}\right\rangle+c_{2} \delta\left\langle r^{4}\right\rangle+c_{3} \delta\left\langle r^{6}\right\rangle+\ldots\right] \\
& =F_{i} \delta\left\langle r^{2}\right\rangle \cdot K
\end{aligned}
$$

where $c_{i}$ are proportional to the expansion coefficients of the difference of the electronic charge density. All relations $c_{2} / c_{1}$, and $c_{3} / c_{1}$ are equal (within a fraction of a percent $)$ for all transitions. $\left(c_{2} / c_{1}=\right.$ $-1.19 \cdot 10^{-3} \mathrm{fm}^{-2}, c_{3} / c_{1}=3.66 \cdot 10^{-6} \mathrm{fm}^{-4}$ for Au.) $c_{1}$ is identical with the electronic factor $F_{i}$, which is proportional to the charge density difference at $r=0$; i.e., $c_{1}=F_{i}=\frac{2 \pi}{3} Z e \Delta|\psi(0)|^{2}$. For this element Au the 
Table 2. Electronic factor $F_{i}$ in $\mathrm{GHz} / \mathrm{fm}^{2}$ for various transitions in Au I calculated from single and multi-configuration Dirac-Fock calculations

\begin{tabular}{|c|c|c|c|}
\hline \multirow[t]{2}{*}{ Transition } & \multirow[t]{2}{*}{$\begin{array}{l}\text { Wavelength } \\
(\mathrm{nm})\end{array}$} & \multicolumn{2}{|c|}{$\begin{array}{l}\text { Electronic factors } F_{i} \\
\left(\mathrm{GHz} / \mathrm{fm}^{2}\right)\end{array}$} \\
\hline & & $\begin{array}{l}\text { from } \\
\text { SC-calc. }\end{array}$ & $\begin{array}{l}\text { from } \\
\text { MC-calc. }\end{array}$ \\
\hline $\begin{aligned} 6{ }^{2} S_{1 / 2} & -6{ }^{2} P_{1 / 2} \\
& -7{ }^{2} P_{1 / 2} \\
& -8{ }^{2} P_{1 / 2} \\
\vdots & \\
- & { }^{2} P_{1 / 2}\end{aligned}$ & $\begin{array}{c}267.6 \\
166.5 \\
150.1 \\
\vdots \\
134.4\end{array}$ & $\begin{array}{c}-45.14 \\
-46.23 \\
-46.84 \\
\vdots \\
-47.00\end{array}$ & -43.07 \\
\hline $\begin{aligned} 6{ }^{2} S_{1 / 2} & -6{ }^{2} P_{3 / 2} \\
- & 7^{2} P_{3 / 2} \\
- & { }^{2} P_{3 / 2} \\
\vdots & \\
- & { }^{2} P_{3 / 2}\end{aligned}$ & $\begin{array}{c}242.8 \\
164.6 \\
149.4 \\
\vdots \\
134.4\end{array}$ & $\begin{array}{c}-46.50 \\
-46.90 \\
-46.96 \\
\vdots \\
-47.00\end{array}$ & -42.73 \\
\hline $\begin{aligned} 6{ }^{2} S_{1 / 2} & -7^{2} S_{1 / 2} \\
- & 8^{2} S_{1 / 2} \\
- & 9^{2} S_{1 / 2} \\
- & 10^{2} S_{1 / 2} \\
\vdots & \\
- & { }^{2} S_{1 / 2}\end{aligned}$ & $\begin{array}{c}2 \times 367.0 \\
2 \times 308.9 \\
2 \times 291.1 \\
2 \times 283.1 \\
\vdots \\
2 \times 268.7\end{array}$ & $\begin{array}{c}-41.80 \\
-45.23 \\
-46.16 \\
-45.53 \\
\vdots \\
-47.00\end{array}$ & \\
\hline $\begin{aligned} 6^{2} P_{1 / 2} & -7{ }^{2} S_{1 / 2} \\
& -8^{2} S_{1 / 2} \\
& -9^{2} S_{1 / 2} \\
- & 10^{2} S_{1 / 2}\end{aligned}$ & $\begin{array}{l}583.7 \\
365.1 \\
319.2 \\
300.6\end{array}$ & $\begin{array}{l}+3.34 \\
-0.09 \\
-1.02 \\
-1.39\end{array}$ & \\
\hline $\begin{aligned} 6{ }^{2} P_{3 / 2} & -7{ }^{2} S_{1 / 2} \\
& -8{ }^{2} S_{1 / 2} \\
& -9^{2} S_{1 / 2} \\
- & 10^{2} S_{1 / 2}\end{aligned}$ & $\begin{array}{l}751.1 \\
424.2 \\
363.5 \\
339.5\end{array}$ & $\begin{array}{r}4.70 \\
1.27 \\
0.34 \\
-0.03\end{array}$ & \\
\hline $\begin{aligned} 5^{2} D_{32} & -6{ }^{2} P_{1 / 2} \\
& -6{ }^{2} P_{3 / 2}\end{aligned}$ & $\begin{array}{l}627.8 \\
506.5\end{array}$ & $\begin{array}{l}-111.66 \\
-113.02\end{array}$ & $\begin{array}{l}-105.2 \\
-104.8\end{array}$ \\
\hline $5^{2} D_{5 / 2}-6^{2} P_{3 / 2}$ & 312.3 & -110.60 & -102.4 \\
\hline
\end{tabular}

correction $K$ in Eq. (3), due to the higher moments, is about $8 \%$ (i.e., $K=0.92$ ).

Table 2 shows that the electron charge density at the nucleus of the lower states clearly dominates the magnitude of the $F_{i}$ values. Within each Rydberg series presented here the $F_{i}$ values become more negative, and approach a limiting value which is the corresponding $F_{i}$ value of the ionized system. The higher the Rydberg state the less it contributes to the charge density at the nucleus, and the more the atom can be separated into an ionized core, and a surrounding electron which interacts less and less with this core. That is the reason why the first three series in Table 2 all approach the limiting value $F_{\infty}$ $=-47.0 \mathrm{GHz} / \mathrm{fm}^{2}$, which corresponds to the difference of the charge density between the two ground states of $\mathrm{Au} \mathrm{I}{ }^{2} S_{1 / 2}$, and Au II ${ }^{1} S_{0}$. All other series presented in Table 2, which have an excited state as lower level, show the same behaviour to more negative $F$ values for higher Rydberg states.
An important question is the influence of correlation on the $F_{i}$ values. We have tried to give an answer by performing various MCDF calculations, taking into account the core excitation. A $5 d$-electron excitation should be most preferable from an energetical point of view as its binding energy is only a few eV below the valence $6 s$ electron, whereas the next core level, the $5 p$ shell, is bound already by about $70 \mathrm{eV}$. If one considers only one-particle core excitation contributions into the outer $6 p_{1 / 2}, 6 p_{3 / 2}, 6 d_{3 / 2}$ and $6 d_{5 / 2}$ electronic shells, one arrives at the conclusion that in the case of the lowest ${ }^{2} S,{ }^{2} D$, and ${ }^{2} P$ states a merely reasonable contribution can be expected for the ${ }^{2} P_{3 / 2}$, and ${ }^{2} P_{1 / 2}$ states, only. In these two cases it is possible to construct configuration state functions (CSF) coupled to good angular momentum $J$, and parity, which include $6 s$ one-particle wave functions. As a result the $\mathrm{MCDF}$ wave function for the ${ }^{2} P_{1 / 2}$ state consists of $5 \mathrm{CSF}$ from which the main contributions are as follows

$$
\begin{aligned}
& \left|6^{2} P_{1 / 2}\right\rangle=0.984\left|5 d^{10} 6 p_{1 / 2} ; J=1 / 2\right\rangle \\
& +0.111\left|5 d_{3 / 2}^{3} 5 d_{5 / 2}^{6} 6 s 6 p_{1 / 2} ; J=1 / 2\right\rangle \\
& -0.107\left|5 d_{3 / 2}^{4} 5 d_{5 / 2}^{5} 6 s 6 p_{3 / 2} ; J=1 / 2\right\rangle+\ldots
\end{aligned}
$$

The ${ }^{2} P_{3 / 2}$ state consists of $8 \mathrm{CSF}$ with its main contributions

$$
\begin{aligned}
& \left|6^{2} P_{3 / 2}\right\rangle=0.974\left[5 d^{10} 6 p_{3 / 2} ; J=3 / 2\right\rangle \\
& +0.144\left|5 d_{3 / 2}^{4} 5 d_{5 / 2}^{5} 6 s 6 p_{1 / 2} ; J=3 / 2\right\rangle \\
& +0.134\left|5 d_{3 / 2}^{4} 5 d_{5 / 2}^{5} 6 s 6 p_{3 / 2} ; J=3 / 2\right\rangle+\ldots .
\end{aligned}
$$

The contributions, which include a $6 s$ electron, increase the density at the nucleus.

In the case of the ${ }^{2} S$, and ${ }^{2} D$ states only one-particle core excitations contribute to the MCDF wave function, which do not change the number of $6 \mathrm{~s}$ electrons, and thus the charge density will not be changed noticeably. Instead in the ${ }^{2} D$ states two-particle excitation from $6 s^{2}$ to $6 p^{2}$ (i.e., $6 p_{1 / 2}^{2}, 6 p_{3 / 2}^{2}, 6 p_{1 / 2}^{1}$ $6 p_{3 / 2}^{1}$ ) contribute, which decreases the charge density at the nucleus. Both tables include the results of the MCDF calculations. As it is not possible with the MCDF code to include open shells with the same Dirac $\kappa$ quantum number, and the same number of occupation in the configuration state functions, only those $F_{i}$ values are given in the last column of Table 2 where a calculation was possible.

The overall picture is, nevertheless, that the maximal change is in the order of $5 \%$ in the $F_{i}$ values so that this contribution, due to the influence of correlation, is about as large as the influence due to the change of the electron density over the nuclear volume. However, both corrections go in the same direction. The $F_{i}$ value $-45.14 \mathrm{GHz} / \mathrm{fm}^{2}$, calculated here in a single configuration $a b$-initio way for the resonance 
transition $6 s^{2} S_{1 / 2}-6 p^{2} P_{1 / 2}$, agrees quite well with a semi-empirical estimate of $F_{i}=-50(5) \mathrm{GHz} / \mathrm{fm}^{2}$ [12]. (The multi-configuration value -43.07 $\mathrm{GHz} / \mathrm{fm}^{2}$ is not too far away either.) This semiempirical value has been estimated from the experimental hyperfine structure splitting in the $6 s^{2} S_{1 / 2}$ ground state, and by the Fermi-GoudsmitSegré formula [7]. In addition, the effect of relativity on the hyperfine structure is included by relativistic correction factors tabulated by Kopfermann [7], and on the isotope shift with formulas given by Bodmer [29], and Babushkin [30]. The effect of screening on the $F_{i}$ value was estimated by extrapolating from data for Os [22]. There are, therefore, a number of uncertainties involved in such a semi-empirical procedure. Additionally, it should be pointed out that the hyperfine structure operator, and the field shift operator are quite different relativistically [31], which has not been considered fully in the semi-empirical approach. Our values are evaluated in an $a b$ initio way using the correct form of the field shift operator. The ab-initio values given in Table 2 should, therefore, serve as a guide in the analysis of isotope shift data in other transitions, and for the extraction of $\delta\left\langle r^{2}\right\rangle$ values from such measurements. In the analysis of experimental isotope shifts normally a King plot procedure is adopted. The isotope shifts in a transition $i$ are plotted as a function of the corresponding isotope shifts in another transition $j$, known as the reference. The slope of the resulting line is given as the ratio between changes in the electron density at the nucleus $\Delta \rho_{i} / \Delta \rho_{j}$ or in terms of the factors $F_{i} / F_{j}$ for the two transitions $i$ and $j$. In the earlier analysis of transitions in $\mathrm{BaI}$ and $\mathrm{Ba} I \mathrm{I}$ [15] slopes, evaluated with $\mathrm{MCDF}$ wave functions, were found to describe the experimental values very well. Theoretical values of such slopes, with the $6 s^{2} S_{1 / 2}-6 p^{2} P_{1 / 2}$ transition as reference line, can be calculated easily from the values given in Table 2. Unfortunately, at present no experimental values for comparison exist, however, it is hoped to obtain in the near future from measurements at the ISOLDE [12].

This work was supported financially in part by the Swedish Natural Science Research Council (NFR).

The use of the computer at GSI and Gothenburg University Computing Centre is acknowledged gratefully.

The authors thank Prof. H.-J.Kluge, Dr. H. Kremmling, and Torbjörn Olson for fruitful discussions.

\section{References}

1. Kluge, H.J.: In: Progress in atomic spectroscopy. Hanle, W., Kleinpoppen, H. (eds.), Part B, p. 727. New York, London: Plenum 1979

2. Ekström, C.: Nucl. Instrum. Methods 186, 261 (1981)

3. Thibault, C., Guimbal, P., Klapisch, R., de Saint Simon, M., Serre, J.M., Touchard, F., Büttgenbach, S., Duong, H.T., Ja- quinot, P., Juncar, P., Liberman, S., Pillet, P., Pinard, J., Vialle, J.L., Huber, G., Pesnelle, A.: Nucl. Instrum. Methods 186, $193(1981)$

4. Neugart, R.: Nucl. Instrum. Methods 186, 165 (1981)

5. Stroke, H.J.: In: Atomic physics 8. Proceedings of the Eight International Conference on Atomic Physics, August 2-6, 1982. Lindgren, L., Rosén, A., Svanberg, S. (eds.). New York, London: Plenum Press 1983

6. Ekström, C.: 4th Int. Conf. on Nuclei far from Stability. Helsingør, Denmark, June 7-13, 1981, CERN Report 81-09, p. 12

7. Kopfermann, H.: Nuclear moments. New York: Academic Press 1958

8. Fuller, G.H.: J. Phys. Chem. Ref. Data 5, 835 (1976)

9. Ekström, C., Lindgren, I., Ingelman, S., Olsmats, M., Wannberg, G.: Phys. Lett. 60 B, 146 (1976)

10. Ekström, C., Robertsson, L., Ingelman, S., Wannberg, G., Ragnarsson, I.: Nucl. Phys. A 348, 25 (1980)

11. Childs, W.J., Goodman, L.S.: Phys. Rev. 141, 176 (1966)

12. Schaaf, H.W.: Dissertation, Mainz 1983

13. Kluge, H.-J., Kremmling, H., Schuessler, H.A., Streib, J., Wallmeroth, K.: Z. Phys. A - Atoms and Nuclei 309, 187 (1983)

14. Bollen, G., Kluge, H.-J., Kremmling, H., Schaaf, H., Streib, J., Wallmeroth, K.: In: Laser spectroscopy VI. p. 214. Springer Series in Optical Sciences. Weber, H.P., Luithy, W. (eds.) Berlin, Heideiberg, New York: Springer Verlag 1983

15. Fricke, B., Grundevik, P., Lindgren, I., Olsson, G., Olsson, T., Rosén, A., Torbohm, G.: Phys. Lett. 97 A, 1983 (1983)

16. Shera, E.B., Wohlfart, H.D., Hoehn, M.V.: Phys. Lett. 112 B, 124 (1982)

17. Kunold, W., Schneider, M., Simons, L.M., Wuest, J., Abela, R.: Z. Phys. A - Atoms and Nuclei 313, 11 (1983)

18. Grundevik, P., Lundberg, H., Nilsson, L., Olsson, G.: Z. Phys. A - Atoms and Nuclei 306, 195 (1982)

19. Grundevik, P., Gustavsson, M., Olsson, G., Olsson, T.: Z. Phys. A - Atoms and Nuclei 312, 1 (1983)

20. Fricke, B., Torbohm, G., Rosén, A.: (to be published)

21. Bauche, J., Champeau, R.-J.: Adv. At. Mol. Phys. 12, 39 (1976)

22. Heilig, K., Steudel, A.: At. Data Nucl. Data Tables 14, 613 (1974)

23. Labarthe, J.J.: J. Phys. B: At. Mol. Phys. 6, 1761 (1973)

24. Martensson, A.-M., Salomonson, S.: I. Phys. B: At. Mol. Phys. 15, 2115 (1982)

25. Desclaux, J.P.: Comput. Phys. Commun. 9, 31 (1975)

26. Moore, C.E.: Nat1. Bur. Std. (US), Circ. No. 467. G.P.O., Washington, D.C., 1949

27. Landolt-Börnstein. Zahlenwerte und Funktionen. Hellwege, K.H. (ed.), Bd. 2. Berlin, Heidelberg, New York: Springer 1967

28. Seltzer, E.C.: Phys. Rev. 188, 1916 (1969)

29. Bodmer, A.R.: Proc. Phys. Soc. A 66, 1041 (1953)

30. Babushkin, F.A.: Sov. Phys. JETP 17, 1118 (1963)

31. Bauche, J.: Comm. At. Mol. Phys. 10, 57 (1981)

A. Rosén

Department of Physics

Chalmers University of Technology

S-41296 Göteborg

Sweden

B. Fricke

G. Torbohm

Fachbereich 18 - Physik

Theoretische Physik

Gesamthochschule Kassel

Heinrich-Plett-Strasse

D-3500 Kassel-Oberzwehren

Federal Republic of Germany 